PROCEEDINGS OF THE

AMERICAN MATHEMATICAL SOCIETY

Volume 130, Number 1, Pages 49-52

S 0002-9939(01)06097-X

Article electronically published on May 3, 2001

\title{
ON THE CONNECTIVITY OF THE JULIA SET OF A FINITELY GENERATED RATIONAL SEMIGROUP
}

\author{
YESHUN SUN AND CHUNG-CHUN YANG
}

(Communicated by Linda Keen)

\begin{abstract}
In this paper we show that the Julia set $J(G)$ of a finitely generated rational semigroup $G$ is connected if the union of the Julia sets of generators is contained in a subcontinuum of $J(G)$. Under a nonseparating condition, we prove that the Julia set of a finitely generated polynomial semigroup is connected if its postcritical set is bounded.
\end{abstract}

\section{INTRODUCTION}

Let $S$ denote a Riemann surface and $\mathcal{H}(S)$ the set of nonconstant holomorphic mappings on $S$. Under composition, $\mathcal{H}(S)$ is a semigroup. We study the subsemigroups of $\mathcal{H}(S)$ from the point of view of dynamics. Given a subsemigroup $G$ of $\mathcal{H}(S)$, the domain of normality for $G$ is called the Fatou set $F(G)$, and its complement $S \backslash F(G)$ is called the Julia set $J(G)$. One can think of this as a dichotomy by the dynamical behavior of the semigroup at random. Here, we will deal with the case when $S$ is the Riemann sphere $\hat{\mathbf{C}}$.

By a rational semigroup we mean a subsemigroup $G$ of $\mathcal{H}(\hat{\mathbf{C}})$ containing at least one element with degree $\geq 2$. If a rational semigroup consists only of polynomials, we call it a polynomial semigroup. If a rational semigroup $G$ can be freely generated by its finite elements $\left\{f_{1}, \cdots, f_{n}\right\}$, we write $G=\left\langle f_{1}, \cdots, f_{n}\right\rangle$, where $n$ is a positive integer, and call it a finitely generated rational semigroup.

This setting is a generalization of the study of the iteration of one rational function. As a matter of fact, a principal aim for the study of rational semigroups is to see how far the classical theory of the iteration of a rational function applies in this more general setting. W. Zhou and F. Ren [5] initiated the study of the dynamics of rational semigroups by introducing the concept of random iteration. There have been some basic results along this line. For instance, the closure of the set of repelling periodic points is the Julia set 11.

In this paper, we deal with the connectivity of the Julia set. It is well known that the Julia set of a polynomial is connected if and only if the orbits of its critical points are bounded [2]. In the case of polynomial semigroup, however, we no longer have the corresponding conclusion. As a counterexample, we take $p_{1}(z)=z^{2}+4 z$ and $p_{2}(z)=z^{2}-4 z+4$. Then $J\left(p_{1}\right)=[-4,0]$ and $J\left(p_{2}\right)=[0,4]$.

Received by the editors May 4, 2000.

2000 Mathematics Subject Classification. Primary 37F10, 37F50.

Key words and phrases. Connectivity, Julia set, rational semigroup.

This research was partially supported by a UGC grant of Hong Kong, Project No. 6070/98P. 
Since $J\left(p_{1}\right) \cup J\left(p_{2}\right)=[-4,4]$ is connected, it follows from Theorem 1 that $J\left(\left\langle p_{1}, p_{2}\right\rangle\right)$ is connected (see Figure [1). On the other hand, for the critical point -2 of $p_{1}$, $\left\{p_{2}^{\circ k} \circ p_{1}(-2)\right\}_{k=1}^{\infty}$ is not bounded. Each $p_{2}^{\circ k} \circ p_{1}$ has -2 as its critical point. Therefore, the boundedness of the postcritical orbits set is not necessary for the Julia set to be connected. Nevertheless, it is a sufficient condition as is proved in Theorem 2 below.

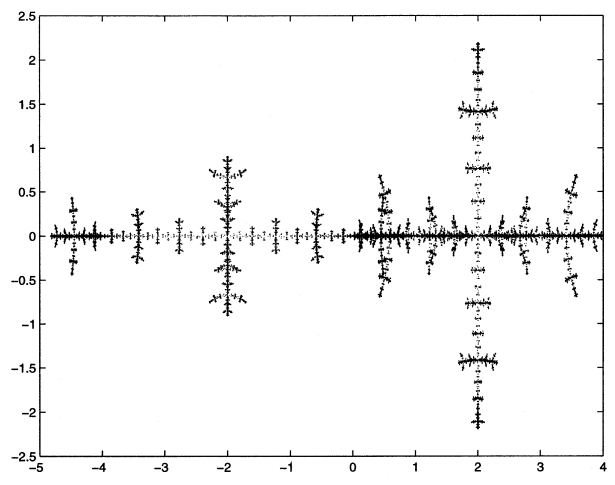

Figure 1. The Julia set $J\left(\left\langle p_{1}, p_{2}\right\rangle\right)$ is connected, where $p_{1}(z)=$ $z^{2}+4 z$ and $p_{2}(z)=z^{2}-4 z+4$.

\section{Preliminaries}

By a continuum, we mean a nonempty compact connected metric space. A subcontinuum is a continuum as a subspace. The following lemma comes from continuum theory (see for example Nadler [3]). This general property will be used in the proof of our main result.

Lemma 1. Let $X$ and $Y$ be two continua and let $f: X \longrightarrow Y$ be an open map. If $B$ is a subcontinuum of $Y$, then each component $A$ of $f^{-1}(B)$ is mapped onto $B$ by $f$.

For simplicity, we follow the definitions in [4].

Definition 1. Let $G$ be a rational semigroup and $z$ a point of $\hat{\mathbf{C}}$. The backward orbit $O^{-}(z)$ of $z$ and the set of exceptional points $E(G)$ are defined by $O^{-}(z)=\{w \in$ $\hat{\mathbf{C}} \mid$ there is some $g \in G$ such that $g(w)=z\}$ and $E(G)=\left\{z \in \hat{\mathbf{C}} \mid \operatorname{CardO}^{-}(z) \leq 2\right\}$ respectively.

Definition 2. Let $G$ be a rational semigroup. The postcritical set $P(G)$ is defined by $P(G)=\overline{\bigcup_{g \in G}\{\text { critical values of } g\}}$. Let $G$ be a polynomial semigroup. The finite postcritical set $P^{*}(G)$ is defined by $P^{*}(G)=P(G) \backslash\{\infty\}$.

We abuse notation and write $F(g)$ for $F(\langle g\rangle)$ and $J(g)$ similarly. The following three lemmas are recalled from Hinkkanen and Martin [1].

Lemma 2. Let $G$ be a rational semigroup. For any $g \in G, g(F(G)) \subset F(G)$, $g^{-1}(J(G)) \subset J(G), F(G) \subset F(g)$, and $J(g) \subset J(G)$.

Lemma 3. Let $G$ be a rational semigroup. Then $E(G)=\left\{z \in \hat{\mathbf{C}} \mid \operatorname{CardO}^{-}(z)<\right.$ $\infty\}$ and $\operatorname{Card} E(G) \leq 2$. 
Lemma 4. Let $G$ be a rational semigroup. If a point $z$ is not in $E(G)$, then $J(G) \subset$ $\overline{O^{-}(z)}$. In particular, taking a point $z \in J(G) \backslash E(G)$, we have $\overline{O^{-}(z)}=J(G)$.

\section{The Connectivity of the Julia Set}

First, we give a general result for the connectivity of the Julia set of a finitely generated rational semigroup.

Theorem 1. Let $G=\left\langle f_{1}, \cdots, f_{n}\right\rangle$ be a finitely generated rational semigroup with $\left\{f_{1}, \cdots, f_{n}\right\}$ as the generator system. We assume that the Julia set of each generator is nonempty. Then the Julia set $J(G)$ is connected if and only if the union of the Julia sets of generators $\bigcup_{i=1}^{n} J\left(f_{i}\right)$ is contained in some subcontinuum $K \subset J(G)$.

Proof. Let $g=f_{i_{1}} \circ \cdots \circ f_{i_{k}}$ be an element in $G$, where $k$ is a positive integer, $i_{j} \in\{1, \cdots, n\}, j=1, \cdots, k$. Define subsets $K_{j}$ inductively as follows. Let $K_{0}=$ $K$. Let $K_{j}=f_{i_{j}}^{-1}\left(K_{j-1}\right) \cup K_{j-1}$ for $j=1, \cdots, k$. Obviously, by Lemma 2 $K \subset K_{j-1} \subset K_{j} \subset J(G)$ for each $j=1, \cdots, k$. Each $K_{j}$ is connected. For if $K_{0}=K$ is connected, we assume $K_{j-1}$ is connected. Since $f_{i_{j}}$ is an open map on $\hat{\mathbf{C}}$, by Lemma 1, each of the finitely many components of $f_{i_{j}}^{-1}\left(K_{j-1}\right)$ is mapped onto $K_{j-1}$. Notice that $J\left(f_{i_{j}}\right) \neq \emptyset$ is in $K_{j-1}$ and completely invariant under $f_{i_{j}}$. Therefore, each component of $f_{i_{j}}^{-1}\left(K_{j-1}\right)$ meets $K_{j-1}$, and $K_{j}$ is hence connected. Specifically, $K_{k}$ is connected.

We take $K_{g}$ to be $K_{k}$ and set $K^{*}$ to be $\bigcup_{g \in G} K_{g}$. Because $K$ is contained in each $K_{g}, K^{*}$ is a connected subset in $J(G)$. There is at least one $f_{i}$ with degree $\geq 2$. Then, $K$ contains at least three points. It follows from Lemma 4 that we can take a point $z$ in $K \backslash E(G)$. From the construction of $K_{g}$, it follows that $g^{-1}(z) \subset K_{g}$. Thus, $O^{-}(z) \subset K^{*}$. Consequently, $J(G)=\overline{O^{-}(z)} \subset \overline{K^{*}} \subset \overline{J(G)}=J(G)$. This implies that $J(G)$ is connected. The proof is complete.

Remark 1. The connectivity of the Julia set of each generator is not required in Theorem 1

As mentioned in Section 1, the Julia set of a finitely generated polynomial semigroup may be connected even though the postcritical set is not bounded. Using Theorem 1 we present the following theorem. Recall that a plane continuum is called nonseparating if its complement in the plane is connected.

Theorem 2. Let $G=\left\langle p_{1}, \cdots, p_{n}\right\rangle$ be a finitely generated polynomial semigroup with the generator system $\left\{p_{1}, \cdots, p_{n}\right\}$ and each generator of degree $\geq 2$. If the finite postcritical set $P^{*}(G)$ is bounded and if we further assume that at least one Julia set is nonseparating, then the Julia set $J(G)$ is connected.

Proof. If $\bigcup_{i=1}^{n} J\left(p_{i}\right)$ is connected, the conclusion follows from Theorem 1 immediately.

We assume that $L=\bigcup_{i=1}^{n} J\left(p_{i}\right)$ is not connected. Since each finite critical orbit of $p_{i}$ is contained in $P^{*}(G)$, each $J\left(p_{i}\right)$ is a continuum. We may assume that $J\left(p_{1}\right)$ is a nonseparating continuum so that $J\left(p_{1}\right)$ is identical with the filled Julia set $K\left(p_{1}\right)$. We suppose that $J\left(p_{1}\right) \cap J\left(p_{i}\right)=\emptyset$. If $J\left(p_{1}\right)$ belongs to the unbounded Fatou component of $p_{i}$, then $K\left(p_{1}\right)$ and $K\left(p_{i}\right)$ are disjoint. Taking a critical point $c$ of $p_{i}$ in $K\left(p_{i}\right)$, we note that $p_{1}^{k}(c) \in P^{*}(G)$ and $\left\{p_{1}^{k}(c)\right\}$ is unbounded since $c \notin K\left(p_{1}\right)$. The contradiction shows that if $J\left(p_{1}\right) \cap J\left(p_{i}\right)=\emptyset$, then $J\left(p_{1}\right)$ lies in some bounded Fatou component $U$ of $p_{i}$. The same argument shows that $P^{*}(G) \subset J\left(p_{1}\right)$ since 
$P^{*}(G)$ is forward invariant under $p_{1}$. In particular, $P^{*}\left(p_{i}\right) \subset J\left(p_{1}\right)$, from which it follows that $p_{i}$ has no Siegel disks and, indeed, that there is just one attracting cycle. In fact it is an attracting fixed point which belongs to $J\left(p_{1}\right) \subset U$. Further, $U$ is the domain of attraction of this fixed point: indeed, $U$ is completely invariant under $p_{i}$.

Further, if $J\left(p_{j}\right)$ meets neither $J\left(p_{1}\right)$ nor $J\left(p_{i}\right)$, then $J\left(p_{1}\right)$ belongs to some Fatou component $V$ of $J\left(p_{j}\right)$. Thus, $J\left(p_{1}\right) \subset U \cap V$ while $\partial U \cap \partial V=\emptyset$. Hence, either $U \subset V$ or $V \subset U$.

Thus, we may find a sequence of integers $i_{1}, i_{2}, \cdots, i_{m}$ and domains $U_{i_{1}}, U_{i_{2}}, \cdots$, $U_{i_{m}}$ such that $\partial U_{i_{j}} \cap \partial U_{i_{j+1}}=\emptyset, J\left(p_{1}\right) \subset U_{i_{1}} \subset U_{i_{2}} \subset \cdots \subset U_{i_{m}}$, where $U_{i_{j}}$ is an invariant Fatou component of $p_{i_{j}}$ and each $J\left(p_{\nu}\right), 1 \leq \nu \leq n$, is in the component of $L$, which contains some $J\left(p_{i_{k}}\right)$.

By the above construction the attracting fixed point $\alpha$ of $p_{i_{k}}$ in $U_{i_{k}}$ lies in $J\left(p_{1}\right)$ as do all the singularities of $p_{i_{k}}^{-n}$. Thus, for any $n \in \mathbf{N}, K_{n}=p_{i_{k}}^{-n}\left(J\left(p_{1}\right)\right)$ is connected

and contains $\alpha$, so that the closed set $K(k)=\overline{\bigcup_{n=0}^{\infty} K_{n}}$ is connected. It also meets $J\left(p_{i_{k}}\right)$. Hence, $\left(\bigcup_{\nu=1}^{n} J\left(p_{\nu}\right)\right) \cup\left(\bigcup_{k=1}^{m} K(k)\right)$ is a connected subset of $J(G)$, using Lemma 2, It follows from Theorem [1] that $J(G)$ is connected.

Remark 2. One cannot omit the nonseparating condition. For instance, we let $f_{1}(z)=z^{2}$ and $f_{2}(z)=z^{3} / 4$. The Julia set $J\left(\left\langle f_{1}, f_{2}\right\rangle\right)$ is not connected.

\section{ACKNOWLEDGEMENT}

The authors thank the referee for an especially careful reading of this paper and for the modifying of the original proof of Theorem 2

\section{REFERENCES}

[1] A. Hinkkanen and G. J. Martin, The dynamics of semigroups of rational functions I, Proc. London Math. Soc. 73(1996), 358-384. MR 97e:58198

[2] J. Milnor, Dynamics in one complex variable: Introductory lectures, Vieweg Verlag, 1999. CMP 2000:03

[3] S. B. Nadler, Continuum Theory: An introduction, Marcel Dekker, 1992. MR 93m:54002

[4] H. Sumi, On dynamics of hyperbolic rational semigroups, J. Math. Kyoto Univ. 37(1997), 717-733. MR 99h:30028

[5] W. Zhou and F. Ren, The Julia sets of the random iteration of rational functions, Chinese Sci. Bull. 37(1992), 969-971.

Department of Mathematics, The Hong Kong University of Science and Technology, Clear Water Bay, Kowloon, Hong Kong, China

E-mail address: maysun@ust.hk

Current address: Department of Mathematics, Zhejiang University, Hangzhou, Zhejiang 310027, Peoples' Republic of China

E-mail address: sun@math.zju.edu.cn

Department of Mathematics, The Hong Kong University of Science and Technology, Clear Water Bay, Kowloon, Hong Kong, China

E-mail address: mayang@ust.hk 